\title{
Effective Attenuation of Adenosine A1R Signaling by Neurabin Requires Oligomerization of Neurabin
}

\author{
Yunjia Chen, Christopher Booth, Hongxia Wang, Raymond X. Wang, Dimitra Terzi, \\ Venetia Zachariou, Kai Jiao, Jin Zhang, and Qin Wang \\ Departments of Cell, Developmental, and Integrative Biology (Y.C., H.W., R.X.W., Q.W.) and Genetics (K.J.), University of \\ Alabama, Birmingham, Alabama; Department of Pharmacology, University of California, San Diego, California (C.B., J.Z.); and \\ Department of Neuroscience, Friedman Brain Institute, Mount Sinai School of Medicine, New York, New York (D.T., V.Z.)
}

Received May 23, 2017; accepted September 25, 2017

\section{ABSTRACT}

The adenosine $A 1$ receptor $(A 1 R)$ is a key mediator of the neuroprotective effect by endogenous adenosine. Yet targeting this receptor for neuroprotection is challenging due to its broad expression throughout the body. A mechanistic understanding of the regulation of $A 1 R$ signaling is necessary for the future design of therapeutic agents that can selectively enhance A1R-mediated responses in the nervous system. In this study, we demonstrate that A1R activation leads to a sustained localization of regulator of $G$ protein signaling 4 (RGS4) at the plasma membrane, a process that requires neurabin (a neural tissue-specific protein). A1R and RGS4 interact with the overlapping regions of neurabin. In addition, neurabin domains required for oligomerization are essential for formation of the A1R/neurabin/RGS4 ternary complex, as well as for stable localization of RGS4 at the plasma membrane and attenuation of $A 1 R$ signaling. Thus, $A 1 R$ and RGS4 each likely interact with one neurabin molecule in a neurabin homo-oligomer to form a ternary complex, representing a novel mode of regulation of $\mathrm{G}$ protein-coupled receptor signaling by scaffolding proteins. Our mechanistic analysis of neurabin-mediated regulation of A1R signaling in this study will be valuable for the future design of therapeutic agents that can selectively enhance A1R-mediated responses in the nervous system.

\section{Introduction}

Endogenous adenosine is a potent anticonvulsant agent that protects against various excitatory insults via activation of the adenosine A1 receptor (A1R) (Haas and Selbach, 2000; Cunha, 2001; Dunwiddie and Masino, 2001; Stone, 2002; Boison, 2008; Sebastião and Ribeiro, 2009). A1R belongs to the G proteincoupled receptor (GPCR) superfamily and couples to the Gi/o subfamily of heterotrimeric G proteins. Activation of A1R inhibits cAMP production and voltage-gated calcium channels and activates $\mathrm{G}$ protein-operated inwardly rectifying potassium channels. Consequently, A1R activation reduces neurotransmitter release from presynaptic terminals and provokes hyperpolarization of postsynaptic membranes (Haas and Selbach, 2000; Cunha, 2001; Dunwiddie and Masino, 2001; Sebastião and Ribeiro, 2009). Despite its potential therapeutic effectiveness, targeting A1R for neuroprotection has been particularly challenging due to its broad expression in multiple peripheral tissues, including the heart, lung, and kidney (Stone, 2002), which leads to undesired peripheral actions that result in dramatic side effects. A better understanding of the regulatory mechanism governing $\mathrm{A} 1 \mathrm{R}$ signaling and activity is

This work was supported by the National Institutes of Health National Institute of Mental Health [Grant R01MH081917].

https://doi.org/10.1124/mol.117.109462. essential for development of novel strategies targeting this receptor for treatment of neurologic diseases.

One important modulator of $\mathrm{G}$ protein signaling is the family of regulator of $\mathrm{G}$ protein signaling (RGS) proteins, which accelerate hydrolysis of GTP by the G $\alpha$ subunit of the G protein, thus terminating GPCR signaling (Ross and Wilkie, 2000; Hollinger and Hepler, 2002; Neubig and Siderovski, 2002; Neitzel and Hepler, 2006; Xie and Palmer, 2007; Sjögren and Neubig, 2010; Kach et al., 2012). A role for endogenous RGS proteins in regulating A1R signaling has been indicated by enhanced A1R-mediated responses in cells expressing an RGSinsensitive mutant form of Gi or Go protein (Chen and Lambert, 2000; Fu et al., 2006). We previously discovered that RGS4 attenuates A1R signaling, and this effect relies on a neural tissue-specific scaffolding protein, neurabin (Chen et al., 2012). Significantly, neurabin deficiency enhances A1R signaling and promotes the protective effect of adenosine against excitotoxic seizure, suggesting the A1R-neurabin interaction as a valid therapeutic target for specifically enhancing the anticonvulsant effects of endogenous adenosine. Neurabin is a multidomain protein containing (from the $\mathrm{N}$ to $\mathrm{C}$ terminus) an actin-binding domain, a protein phosphatase 1 binding and regulatory sequence, a protein-interaction PDZ domain, three coiled-coil domains, and a putative protein-interaction sterile $\alpha$ motif (SAM) domain (Nakanishi et al., 1997; Burnett et al., 1998).

ABBREVIATIONS: A1R, adenosine A1 receptor; BSA, bovine serum albumin; CFP, cyan fluorescent protein; CHO, Chinese hamster ovary; co-IP, coimmunoprecipitation; GPCR, G protein-coupled receptor; GSH, glutathione; GST, glutathione S-transferase; HA, hemagglutinin; HEK293, human

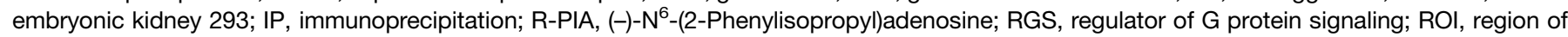
interest; SAM, sterile $\alpha$ motif; TIRF, total internal reflection fluorescence; YFP, yellow fluorescent protein. 
Sequences that are responsible for its interaction with $\mathrm{A} 1 \mathrm{R}$ and RGS4 remain to be determined. In addition, whether and how other functional domains may contribute to the regulation of A1R signaling has not yet been investigated. This information is not only important for mechanistic understanding of neurabindependent regulation of GPCR signaling, but it is also necessary for the future design of therapeutic agents that can selectively enhance A1R-mediated responses in the nervous system.

In this study, we elected to tease out the molecular mechanism by which neurabin fine-tunes A1R signaling using combined genetic, biochemical, and live-cell imaging approaches. Our livecell imaging studies reveal, to our knowledge for the first time, that A1R activation induces a long-lasting localization of RGS4 at the plasma membrane, which requires the presence of neurabin. Our structure-function analysis suggests that the A1R/neurabin/RGS4 ternary complex formation relies on neurabin oligomerization, which is also essential for stable membrane localization of RGS4 and attenuation of A1R signaling.

\section{Materials and Methods}

Heterologous Cell Culture. Human embryonic 293 (HEK293) and Cos7 cells were cultured in Dulbecco's modified Eagle's medium (Invitrogen, Carlsbad, CA) supplemented with $10 \%$ fetal bovine serum (Atlanta Biologicals, Atlanta, GA) and 1\% penicillin/streptomycin and were maintained in a humidified $5 \% \mathrm{CO}_{2}$ incubator. Chinese hamster ovary (CHO)-K1 cells were cultured in Dulbecco's modified Eagle's medium/F-12 (Invitrogen) with 10\% fetal bovine serum, 1\% penicillin/streptomycin, and $2 \mathrm{mM}$ glutamine. Cells were transfected with Lipofectamine 2000 (Invitrogen).

DNA Constructs. cDNAs encoding A1R amino acids 202-326 and RGS4 were amplified by polymerase chain reaction and cloned into the pCMVTNT vector (Promega, Madison, WI). cDNAs encoding different regions of neurabin sequences were amplified by polymerase chain reaction and cloned into the glutathione $S$-transferase (GST) fusion vector pGEX4T (GE Healthcare, Chicago, IL) through EcoRI and XhoI sites to generate the GST-fused Nrb constructs described in Fig. 3 . The GST-Nrb $\Delta 404-413$ construct and GST-Nrb331-453 with mutations were constructed from the GST-Nrb331-453 construct using the QuikChange kit (Stratagene, San Diego, CA). For eukaryotic expression of GST-RGS4 fusion protein, RGS4 cDNA was cloned into a pCMV-GST vector (Sigma, St. Louis, MO).

In Vitro GST Pull-Down Assays. $\left[{ }^{35} \mathrm{~S}\right]$-labeled, in vitro-translated A1R202-326 and RGS4 probes were prepared using the TNT rabbit reticulocyte lysate kit (Promega). Preparation of GST-fusion proteins and pull-down assays was described previously (Wang and Limbird, 2002). In brief, $2 \mu \mathrm{g}$ purified GST or GST-fusion protein was incubated with the $\left[{ }^{35} \mathrm{~S}\right]$-labeled probe $(100,000-300,000 \mathrm{cpm})$ in $0.25 \mathrm{ml}$ binding buffer $[50 \mathrm{mM}$ Tris- $\mathrm{HCl}, \mathrm{pH} 7.4,0.05 \%$ Triton $\mathrm{X}-100,10 \%$ glycerol, $0.01 \%$ bovine serum albumin (BSA), $100 \mu \mathrm{M}$ phenylmethylsulfonyl fluoride, $1 \mu \mathrm{g} / \mathrm{ml}$ soybean trypsin inhibitor, $1 \mu \mathrm{g} / \mathrm{ml}$ leupeptin, and $10 \mathrm{U} / \mathrm{ml}$ aprotinin] for 2 hours. Then $25 \mu \mathrm{l}$ glutathione (GSH) agarose was added to this incubation and rotated for an additional 2 hours. In all studies in which GST-fusion proteins were employed, the same SDS-PAGE gel for autoradiography was stained with Coomassie blue to confirm the presence of the GST-fusion proteins at equivalent amounts.

Coimmunoprecipitation Assays. Coimmunoprecipitation (co-IP) assays were performed as described previously (Chen et al., 2012). Briefly, HEK293 cells (plated at $4 \times 10^{6} / 100$-mm dish) were transfected with the desired plasmids. Forty-eight hours after transfection, cells were stimulated with a selective A1R agonist, (-)- $\mathrm{N}^{6}-(2-\mathrm{Phenylisopropyl)}$ adenosine ( $R$-PIA) (Sigma), for 5 minutes. Cells were then lysed in $350 \mu \mathrm{l}$ immunoprecipitation (IP) buffer (10 mM Tris, $1 \%$ NP-40, $10 \%$ glycerol, $5 \mathrm{mM}$ EDTA, and 5 mM EGTA, pH 7.6, plus protease inhibitors) and incubated with an antibody against hemagglutinin
(HA) (rat anti-HA, 1:100; Roche, Indianapolis, IN), Myc (1:100; Cell Signaling Technology, Danvers, MA), or RGS4 (1:50; Santa Cruz Biotechnology, Dallas, TX) overnight at $4^{\circ} \mathrm{C}$, followed by an additional 2-hour incubation with $30 \mu \mathrm{l}$ protein $\mathrm{G}$ bead slurry (Pierce, Waltham, MA) at $4^{\circ} \mathrm{C}$. Proteins in the IP complex or total lysates were detected by Western blot. HA-A1R was detected by mouse HA.11 antibody followed by a crossadsorbed anti-mouse secondary antibody.

For sequential GST pull-down co-IP experiments, HEK293 cells were plated on $100-\mathrm{mm}$ dishes at $4 \times 10^{6} /$ dish and were transfected with HA-A1R, Myc-Nrb, and GST or GST-RGS4 16 hours later (at 1, 7, and $2 \mu \mathrm{g}$, respectively). Forty-eight hours after transfection, cells were stimulated and lysed in $350 \mu \mathrm{l}$ IP buffer. Cell lysates were first incubated with $30 \mu \mathrm{l}$ GSH agarose slurry (GE Healthcare Life Science) for 2 hours at $4^{\circ} \mathrm{C}$. The bound GST-fusion proteins were then eluted with $200 \mu \mathrm{l}$ free GSH (Sigma) at $25 \mathrm{mM}$ dissolved in IP buffer. The GSH eluates were then incubated with rat anti-HA antibody (1:100 dilution) overnight at $4^{\circ} \mathrm{C}$ followed by an additional 2-hour incubation with $20 \mu \mathrm{l}$ protein $\mathrm{G}$ bead slurry (Pierce) at $4^{\circ} \mathrm{C}$. Quantitation of Western blots was performed using the LI-COR Odyssey imaging system (Lincoln, NE).

Measurement of cAMP Levels. cAMP assays were performed using the AlphaScreen Assay Kit (PerkinElmer, Waltham, MA). In brief, CHO-K1 cells were cotransfected with cDNAs encoding HA-A1R together with Myc-Nrb or Myc-Nrb1-597. Forty-eight hours after transfection, cells were collected in phosphate-buffered saline. The pellet was then resuspended with the stimulation buffer $(1 \times$ Hanks' balanced salt solution, 0.1\% BSA, $0.5 \mathrm{mM}$ 3-isobutyl-1-methylxanthine, and $5 \mathrm{mM}$ HEPES, $\mathrm{pH}$ 7.4) and mixed with anti-cAMP acceptor beads. The mix was divided into three groups for the following treatments: 1) vehicle, 2) $10 \mu \mathrm{M}$ forskolin (Sigma), and 3) $10 \mu \mathrm{M}$ forskolin and $5 \mu \mathrm{M} R$-PIA. Twenty minutes after stimulation at $37^{\circ} \mathrm{C}$, biotinylated cAMP/streptavidin donor beads in lysis buffer (0.1\% BSA, $0.3 \%$ Tween-20, and $5 \mathrm{mM}$ HEPES, $\mathrm{pH}$ 7.4) were added to the cell/acceptor bead mix. After 30-minute incubation at room temperature, fluorescence intensity was analyzed on a BioTek Synergy 2 plate reader (BioTex, Winooski, VT) using standard $\alpha$-screen settings.

Live Imaging of RGS4 Translocation. Cos7 cells were transfected with cDNA encoding A1R-cyan fluorescent protein (CFP) and RGS4-yellow fluorescent protein (YFP) with either full-length neurabin or Nrb1-597 for 48 hours. Cells were serum starved for 24 hours before imaging. Cells were also pretreated with adenosine deaminase (5 U/ml; Sigma) or $1 \mu \mathrm{M}$ 8-cyclopentyl-1,3-dipropylxanthine (Sigma) overnight to eliminate adenosine in the medium before imaging. Live-cell CFP and YFP images were taken at 30-second intervals on a Zeiss Observer Z1 epifluorescent microscope (Carl Zeiss, Jena, Germany) equipped with an Evolve EMCCD camera (Photometrics, Tucson, AZ). Cells were stimulated with $1 \mu \mathrm{M} R$-PIA. Translocation of RGS4 for each cell was measured using a ratio of different plasma membrane regions of interest (ROIs) to a single cytoplasmic reference ROI (ROI ratio). The translocation responses were plotted as individual traces for each plasma membrane ROI. The responses were normalized to the frame prior to drug addition $(t=0)$. Translocation measurements were plotted using GraphPad Prism software (GraphPad Software Inc., La Jolla, CA). Quantification was performed by comparing the slopes of the responses after the initial maximum response was reached. The slope of the response was measured by determining the initial maximum response and calculating the slope of the response from this point through the end of the acquisition. The slopes for each experimental group were averaged and graphed as a box-and-whisker plot.

Live-Cell Total Internal Reflection Fluorescence Imaging of RGS4 Translocation. Cos7 or HEK293T cells were cotransfected with cDNA encoding A1R-CFP, RGS4-YFP, and neurabin (wild type or mutant) for 24-48 hours with Polyjet (SignaGen, Rockville, MD). Control cells were only transfected with A1R-CFP and RGS4-YFP. Cells were serum starved and pretreated with $1 \mu \mathrm{M}$ 8-cyclopentyl1,3-dipropylxanthine overnight before imaging. Live-cell total internal reflection fluorescence (TIRF) images were taken for YFP at 
30 -second intervals on an Olympus IX83 microscope with the CellTIRF4-Line system (Olympus, Tokyo, Japan) and an Andor iXon Ultra EMCCD camera (Andor, Belfast, UK). For YFP excitation, a 491-nm cell* Laser (Olympus) was used and all images were taken with an $\times 150$ TIRF objective (Olympus). CFP imaging was only performed during sample focusing to confirm the presence of A1R in the cells being imaged for the time lapse. For CFP excitation, a 405-nm OBIS laser was used (Coherent Inc., Santa Clara, CA). To stimulate the translocation, $1 \mu \mathrm{M} R$-PIA was added to the cells. Translocation for each cell was determined by taking an ROI of the cell and measuring intensity averages over time. The translocation responses for each cell in each experimental group were plotted as an average trace with S.E.M. bars. The responses were normalized to the frame prior to drug addition $(t=0)$. Quantification was performed by comparing the slopes of the responses after the initial maximum response was reached. The slope of the response was measured by determining the initial maximum response and calculating the slope of the response from this point through the end of the acquisition. The slopes for each experimental group were averaged and graphed as a boxand-whisker plot. The slopes were also statistically compared using an unpaired $t$ test with a 99\% confidence level. Translocation measurements were plotted and statistically analyzed using GraphPad Prism software.

Statistical Analysis. All statistical analyses were performed using GraphPad Prism software. Data were analyzed with twotailed $t$ tests. $P<0.05$ was considered statistically significant.

\section{Results}

Neurabin Is Required for Prolonged Localization of RGS4 on the Plasma Membrane after A1R Stimulation. RGS4 is mainly localized in the cytosol. To turn off the longlasting A1R signaling (Ramkumar et al., 1991; Palmer et al., 1996; Wetherington and Lambert, 2002), RGS4 must be localized at the plasma membrane in a prolonged fashion after A1R stimulation. We examined RGS4 localization in live cells before and after A1R stimulation by a selective agonist, $R$-PIA, using TIRF imaging. In cells coexpressing neurabin with A1R and RGS4, a rapid and stable translocation of RGS4 from the cytosol to plasma membrane was observed after $R$-PIA stimulation (Fig. 1A), which lasted the entire recording period (30 minutes; Fig. 1, B and E). However, in cells without neurabin expression, although $R$-PIA was still able to induce a rapid translocation of RGS4 to the plasma membrane, the membrane localization of RGS4 declined over time and returned to basal or near-basal levels by 30 minutes (Fig. 1, $\mathrm{C}$ and F). The recruitment of RGS4 to the plasma membrane in these cells was much more transient compared with that in cells with neurabin expression (Fig. 1, D and G). These data suggest that although neurabin is not required for the initial recruitment of RGS4 to the plasma membrane by A1R activation, neurabin is crucial for stabilizing a prolonged localization of RGS4 at the plasma membrane.

A1R and RGS4 Interact with the Overlapping Regions of Neurabin. Neurabin has multiple functional domains (illustrated in Fig. 2A). We previously defined amino acids 146-453 as the A1R binding region (Chen et al., 2012). Consistently, A1R was readily coimmunoisolated with neurabin amino acids 1-597 (Nrb1-597; Fig. 2, B and F) but not with neurabin amino acids 454-593 (Nrb454-593; Fig. 2C) in cells stimulated with $R$-PIA. Intriguingly, RGS4 was also found to strongly interact with Nrb1-597 (Fig. 2, D and F) but showed no interaction with Nrb454-593 (Fig. 2E) after $R$-PIA stimulation. These data suggest that the interactions of neurabin with A1R and RGS4 both occur within neurabin amino acids 146-453.
We further mapped sequences within neurabin amino acids 146-453 (Nrb146-453) for direct interaction with A1R and RGS4 by generating and analyzing a series of deletion mutations within this region (illustrated in Fig. 3A). In in vitro GST pull-down assays, neurabin amino acids 331-453 (Nrb331-453) directly interacted with both A1R (amino acids 202-326, 3iloop plus C-terminal tail, regions on A1R that are responsible for neurabin interaction) (Fig. 3B) and RGS4 (Fig. 3C), whereas amino acids 146-330 failed to interact with either of these two proteins. Neurabin sequences that interact with A1R were further narrowed down to amino acids 383-413 (Fig. 3B). In particular, neurabin amino acids 404-413 are essential, as deletion of these 10 amino acids abolished the interaction of Nrb331-453 with A1R (Fig. 3B). Similarly, we also detected a direct interaction of Nrb383-413 with RGS4 (Fig. 3C). However, deletion of amino acids 404-413 only slightly reduced Nrb331-453 interaction with RGS4. In addition, Nrb331-403 could still bind to RGS4, albeit to a lower extent compared with Nrb383-413 (Fig. 3C). On the other hand, Nrb331-403 failed to interact with A1R (Fig. 3B). Together, these data suggest that A1R and RGS4 bind to overlapping regions of neurabin (i.e., amino acids 383-413). While A1R binding requires a linear stretch of neurabin sequence (i.e., amino acids 404-413), RGS4 binding likely involves multiple noncontiguous amino acids within neurabin region amino acids 383-413.

Since sequence 404-413 is required for neurabin interaction with A1R, we further analyzed this region. Within this 10-amino-acid region, there are two valines (V404 and V407), which are highly hydrophobic, and three arginines (R406, R408, and R410), which are positively charged. We generated mutant Nrb331-453 constructs with valines and arginines at these positions mutated to alanines, respectively, and tested their interactions with A1R (amino acids 202-326). Mutations at the two valine sites markedly decreased the binding of Nrb331-453 to A1R, whereas mutations at the three arginine sites only had a slight effect (Fig. 3D). These data suggest that the interaction interface between $\mathrm{A} 1 \mathrm{R}$ and neurabin is likely hydrophobic and not influenced much by charges.

Domains of Neurabin That Mediate Oligomerization Are Required for Assembly of the A1R/Neurabin/RGS4 Ternary Complex. Our data shown above suggest that A1R and RGS4 interact with overlapping regions of neurabin. The question then became whether neurabin interacts with A1R and RGS4 sequentially or the three proteins form a triple, or ternary, complex in cells. To address this, we performed sequential GST pull-down and co-IP experiments (procedure illustrated in Fig. 4A). As shown in Fig. 4B, Myc-tagged neurabin (Myc-Nrb), HA-A1R, and GST-RGS4 (but not GST alone) were coisolated in the same complex from the lysates of cells stimulated with $R$-PIA. These data establish the formation of an A1R/neurabin/ RGS4 ternary complex in cells upon agonist stimulation.

Since A1R and RGS4 interact with overlapping regions of neurabin and the three proteins form a ternary complex upon agonist stimulation, we speculate that A1R and RGS4 may not bind to the same neurabin molecule simultaneously. Instead, since neurabin forms homo-oligomers in cells through the coiled-coil and SAM domains (Oliver et al., 2002), A1R and RGS4 may each bind one monomer of neurabin to form the A1R/neurabin/RGS4 ternary complex (illustrated in Fig. 5A). To test this hypothesis, we examined whether neurabin lacking the coiled-coil and SAM domains can still scaffold the A1R-RGS4 interaction. Consistent with formation of 
A

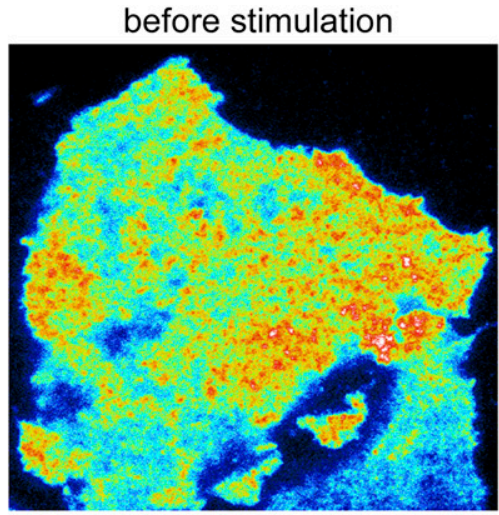

B

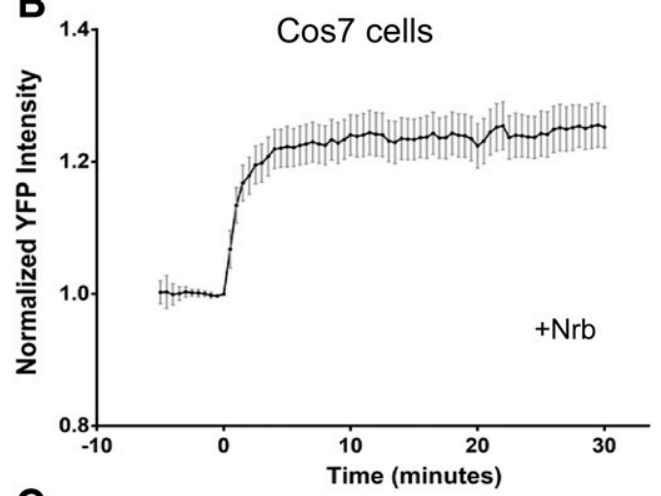

C

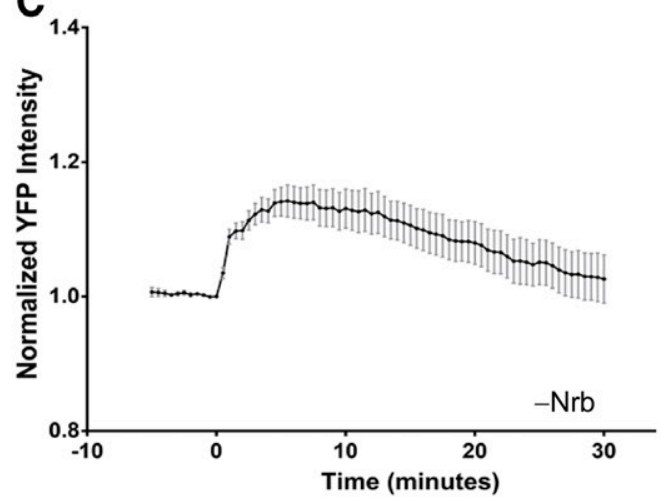

D

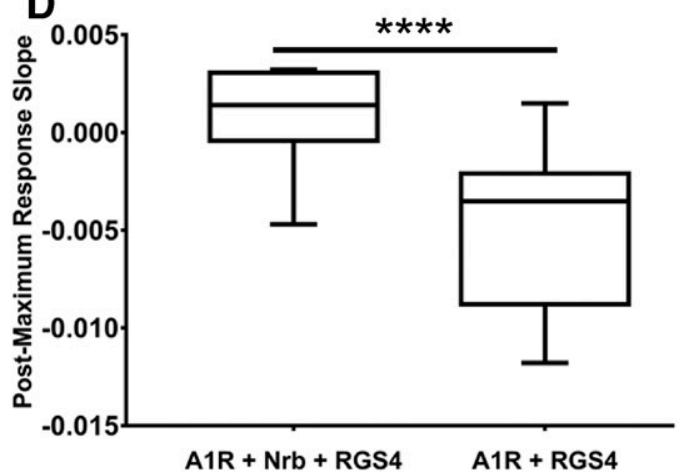

after stimulation

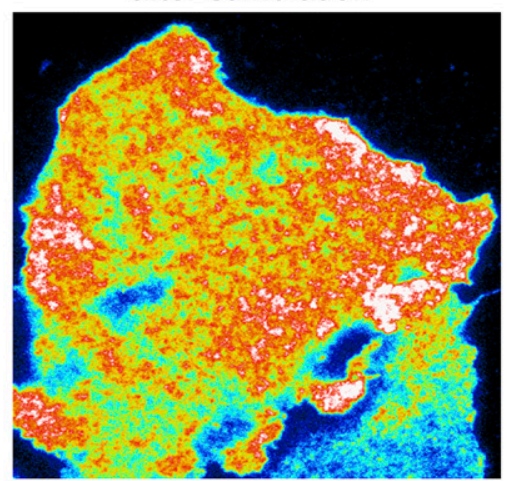

E

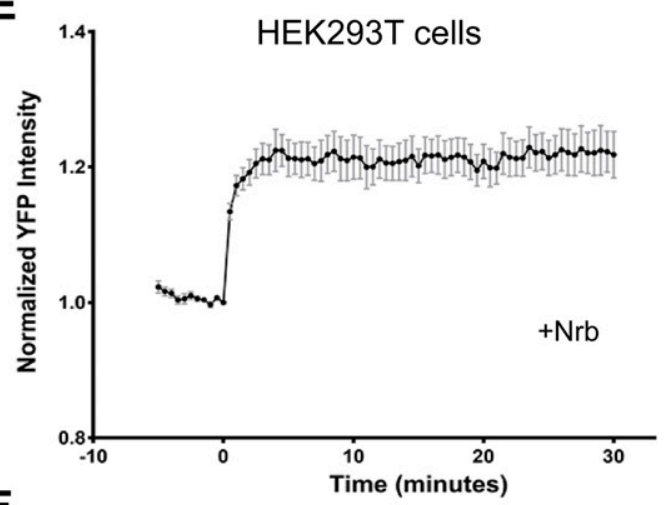

F

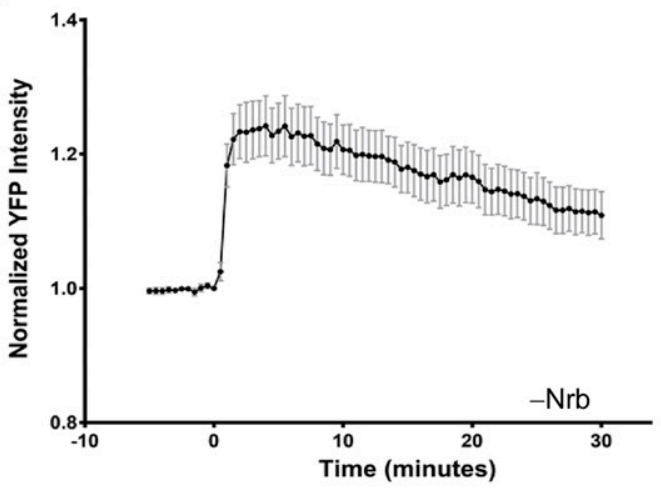

G

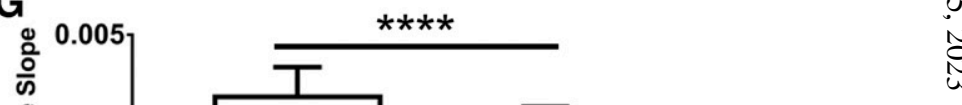

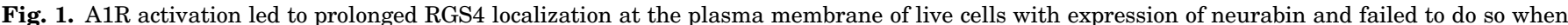

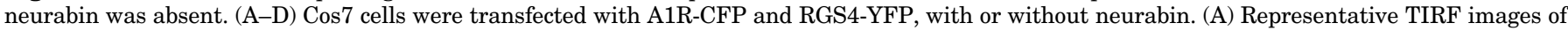

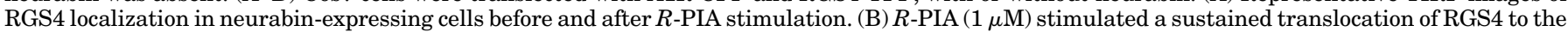

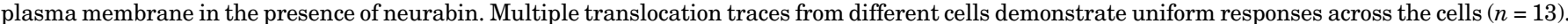

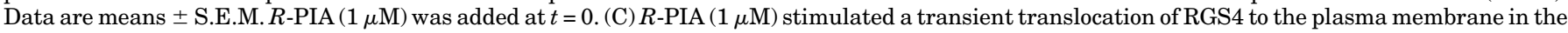

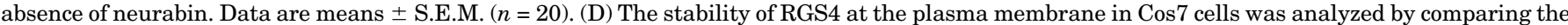

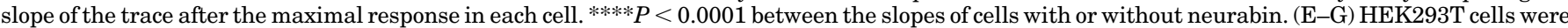

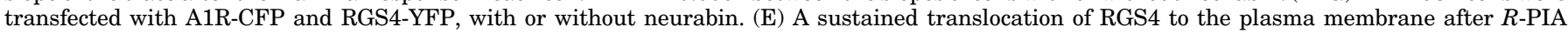



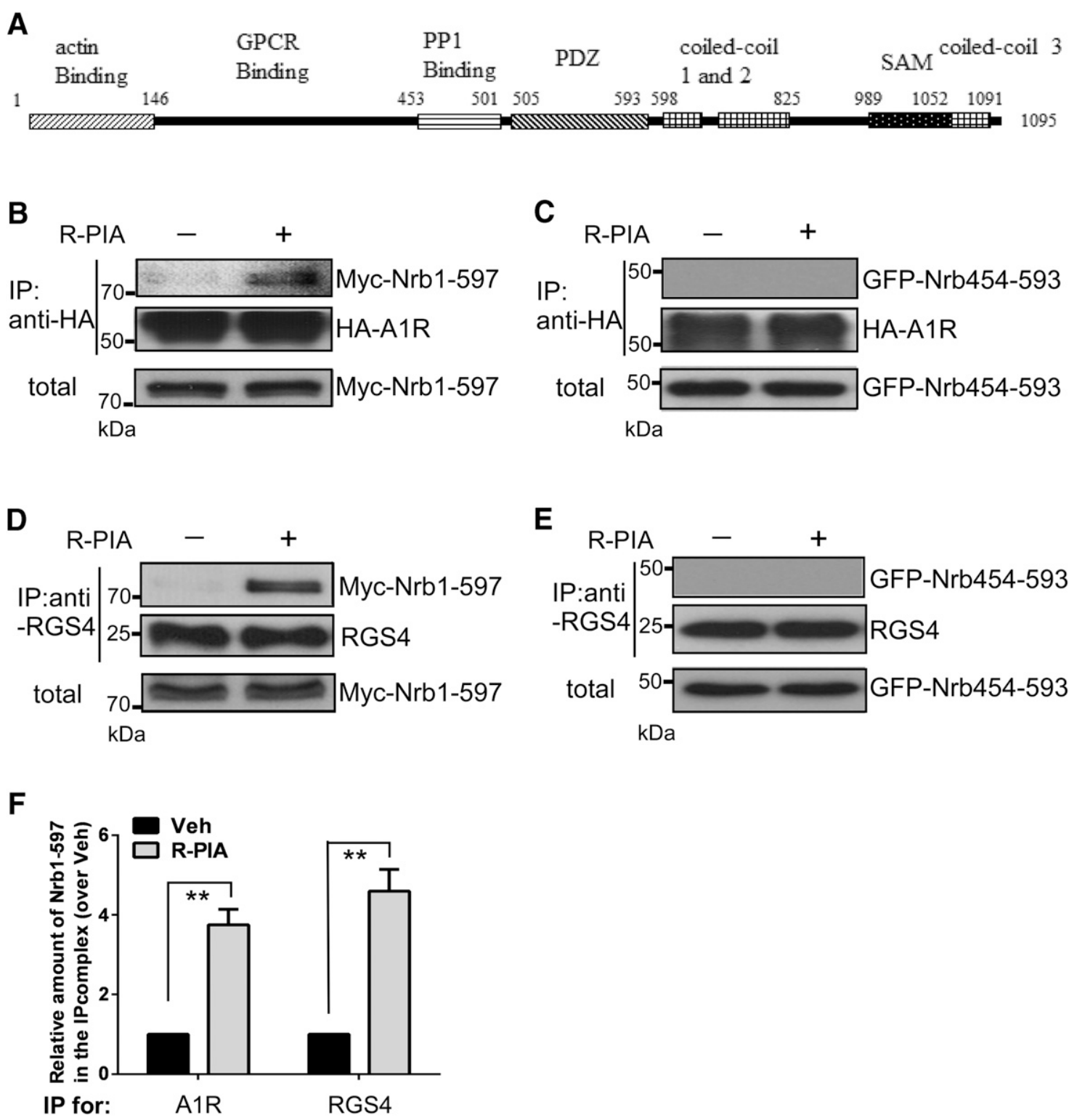

Fig. 2. Neurabin animo acids146-453 are involved in interaction with both A1R and RGS4 in cells. (A) Scheme of neurabin structural domains. (B) HEK293 cells cotransfected with cDNAs encoding HA-A1R and Myc-Nrb1-597 were stimulated with vehicle or $R$-PIA (1 $\mu$ M, 5 minutes) and then lysed and subjected to the IP assay using an HA antibody. (C) HEK293 cells coexpressing HA-A1R with GFP-Nrb454-593 were stimulated and subjected to the IP assay using an HA antibody. (D) HEK293 cells cotransfected with cDNAs encoding HA-A1R, Myc-Nrb1-597, and RGS4 were stimulated with vehicle or $R$-PIA ( $1 \mu \mathrm{M}, 5$ minutes) and then lysed and subjected to the IP assay using an anti-RGS4 antibody. (E) HEK293 cells coexpressing HA-A1R, GFP-Nrb454-593, and RGS4 were stimulated and subjected to the IP assay using an anti-RGS4 antibody. Blots representative of at least three independent experiments are shown. (F) Quantitation of Nrb1-597 interactions with A1R or RGS4 ( $n=4$ for each condition). Data are expressed as the fold change of Nrb1-597 in complex with A1R or RGS4 over the no-stimulation control (defined as 1.0-fold). Values shown are means \pm S.E.M. $* * P<0.01$ $(R$-PIA stimulated vs. control). Veh, vehicle.

neurabin homo-oligomers (Nakanishi et al., 1997; MacMillan et al., 1999; Oliver et al., 2002), we readily detected interaction between Myc and GFP-tagged full-length neurabin (Fig. 5B). Myc-Nrb1-597, in which the coiled-coil and SAM domains are deleted, failed to interact with GFP-tagged full-length neurabin (Fig. 5B). In cells expressing the full-length Myc-Nrb, we detected stable interaction between A1R and RGS4 (Fig. 5C).
However, in cells expressing Nrb1-597, we were unable to coimmunoisolate RGS4 in the same complex with A1R, despite the fact that this neurabin truncation itself can be coimmunoisolated with A1R (Fig. 5C). Together with the fact that Nrb1-597 is able to interact with A1R and RGS4 individually (Fig. 2, B and D), these data suggest that domains mediating neurabin oligomerization are required for assembly

stimulation in the presence of neurabin. Data are means \pm S.E.M. $(n=14)$. $R$-PIA was added at $t=0$. (F) A transient translocation of RGS4 to the plasma membrane in the absence of neurabin. Data are means \pm S.E.M. $(n=16)$. (G) The stability of RGS4 at the plasma membrane in HEK293T cells was analyzed by comparing the slope of the trace after the maximal response in each cell. $* * * * P<0.0001$ between the slopes of cells with or without neurabin. 
A

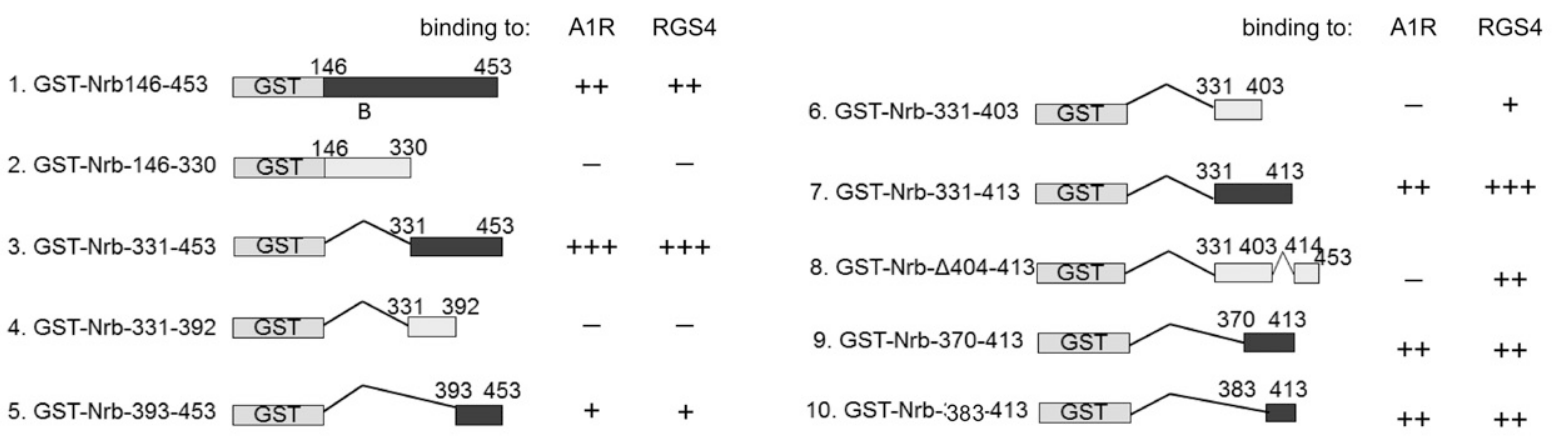

B

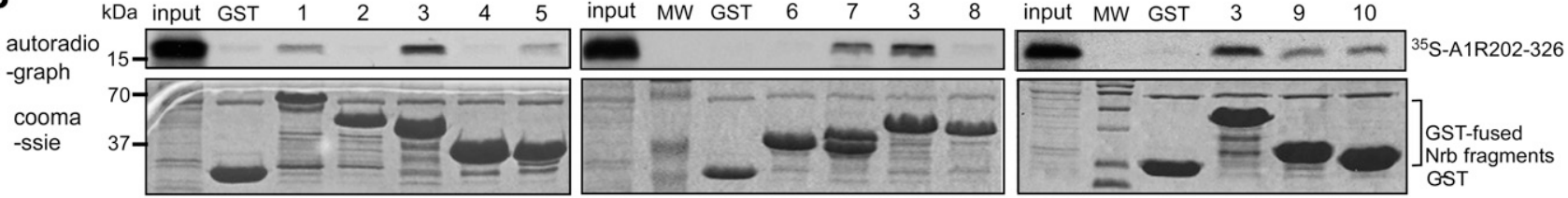

C
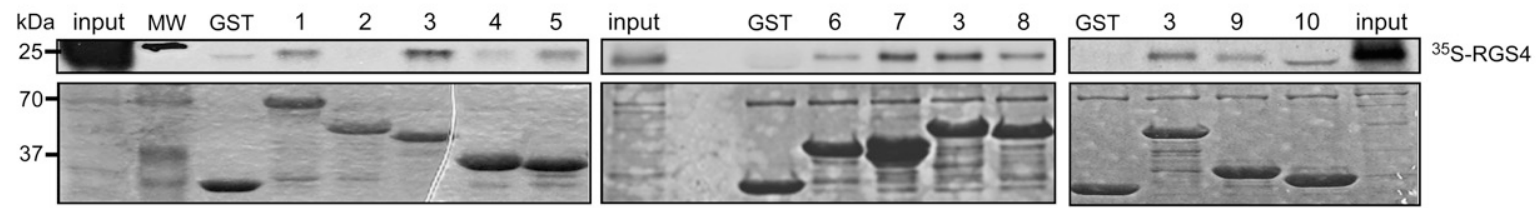

D

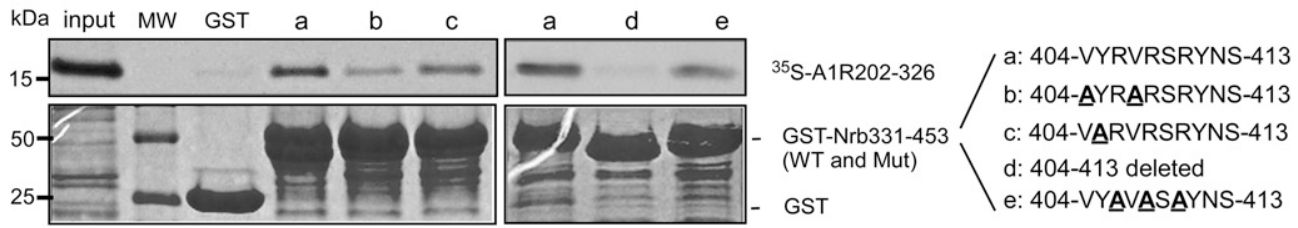

Fig. 3. Binding to A1R and RGS4 involves overlapping sequences within neurabin amino acids 146-453. (A) Schematic diagram of GST-fused wild-type and mutant neurabin amino acids 146-453 with deletions or truncations. Estimated binding strength of each structure to the A1R 3iloop or RGS4, as revealed by in vitro GST pull-down assays, is indicated. (B) In vitro GST pull-down assays with ${ }^{35}$ S-labeled A1R 3iloop and C-tail (amino acids 202-326) as a probe. Bound [ ${ }^{35}$ S]A1R202-326 was detected by autoradiograph. GST and GST-fused neurabin fragments were detected by Coomassie staining. Lanes are numbered as corresponding to structures shown in (A). Input probe represents one-tenth of the total input in each reaction. (C) In vitro GST pull-down assays with ${ }^{35}$ S-labeled RGS4 as a probe. Bound ${ }^{35}$ S]RGS4 was detected by autoradiography. GST and GST-fused neurabin fragments [numbered corresponding to structures shown in (A)] were detected by Coomassie staining. The input probe on the left and right represents one-fifth of the total input in each reaction, whereas the input probe in the middle represents $1 / 30$ of the total input in the reaction. (D) In vitro GST pull-down assays testing interaction between ${ }^{35}$ S-labeled A1R202-326 and GST-Nrb331-453 with indicated mutations within neurabin amino acids 404-413. Bound $\left[{ }^{35}\right.$ S $]$ A1R202-326 was detected by autoradiography. The input probe represents $1 / 20$ of the total input in each reaction. Images are representative of at least three experiments for each pull-down assay. Mut, mutant; MW, molecular weight marker; WT, wild type.

of the A1R/neurabin/RGS4 ternary complex and they support the interaction model illustrated in Fig. 5A.

The Neurabin Oligomerization Domains Are Required for Stabilizing RGS4 on the Cell Surface and Attenuating A1R Signaling. Next we examined the importance of neurabin oligomerization domains in regulating RGS4 translocation in live cells. Consistent with our data shown in Fig. 1, in cells expressing the full-length neurabin, A1R activation with $R$-PIA led to a rapid and long-lasting redistribution of RGS4 to the plasma membrane (Fig. 6, A and B). RGS4 stayed associated with the plasma membrane for the entire recording period up to 60 minutes (Fig. 6D). On the other hand, in cells expressing Nrb1-597, RGS4 translocation to the cell surface was rather transient (Fig. 6C); plasma membrane association of RGS4 started to decline within 20 minutes after stimulation in most cells (Fig. 6, C and E). These data suggest that the neurabin oligomerization domains are crucial for the sustained plasma membrane localization of RGS4 induced by A1R stimulation.

Since Nrb1-597 cannot stabilize RGS4 at the plasma membrane (Fig. 6, C and E) and it cannot scaffold the interaction between A1R and RGS4 (Fig. 5C), we predicted that expression of this mutant form of neurabin would not be able to attenuate A1R signaling in cells. We measured A1Rmediated inhibition of cAMP production stimulated by $R$-PIA in CHO-K1 cells, in which endogenous RGS4 is required for neurabin-mediated attenuation of A1R signaling (Chen et al., 2014). In cells expressing the full-length neurabin, A1Rmediated inhibition of cAMP production was abolished (Fig. 7A). However, expression of Nrb1-597 showed a negligible effect on A1R-mediated cAMP inhibition (Fig. 7A). Expression of either the full-length neurabin or Nrb1-597 had no effect on the A1R expression level (Fig. 7B). These data suggest that domains mediating neurabin oligomerization are required for attenuation of A1R signaling.

\section{Discussion}

In this study, we demonstrate that in the presence of neurabin, A1R activation is able to induce prolonged localization of RGS4 on the plasma membrane (Figs. 1 and 6B). Both 
A

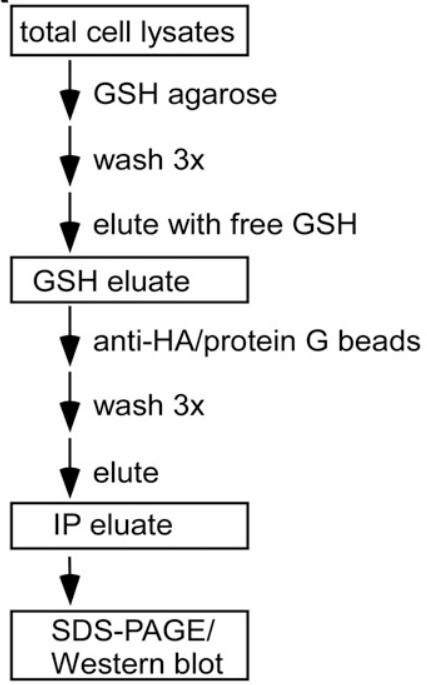

B

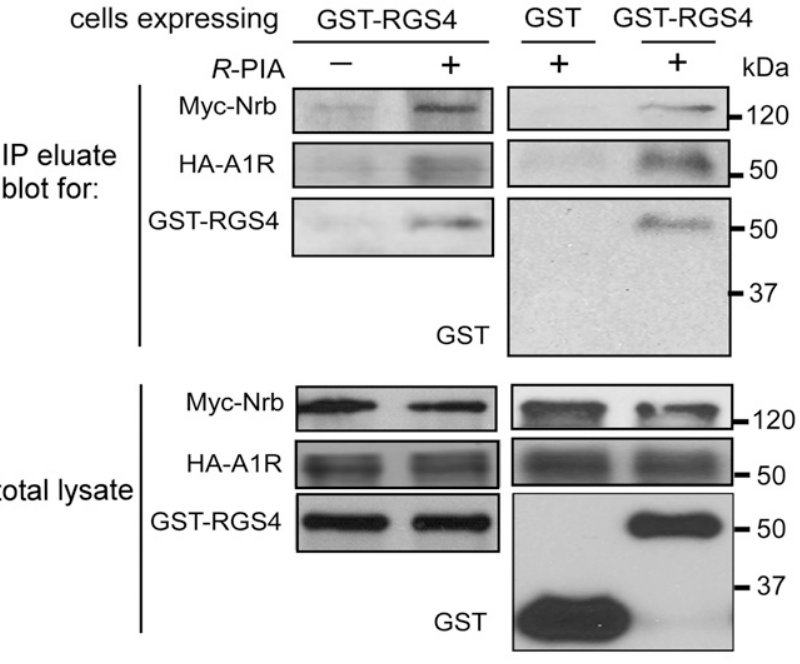

Fig. 4. Neurabin forms a triple complex with A1R and RGS4 through oligomerization. (A) Flowchart of the sequential pull-down and IP procedure. (B) Formation of a neurabin/A1R/ RGS4 ternary complex. HEK293 cells coexpressing Myc-Nrb and HA-A1R, together with GST or GST-RGS4, were stimulated with vehicle or $R$-PIA $(1 \mu \mathrm{M})$ for 5 minutes. Cell lysates were subjected to sequential GST pull-down and co-IP. Blots representative of three independent experiments showing A1R, neurabin, and RGS4 in total lysates and final eluate after sequential pull-down and co-IP.
A1R and RGS4 interact with neurabin amino acids 146-453 in cells (Fig. 2). Further characterization of the interaction between neurabin and $\mathrm{A} 1 \mathrm{R}$ and between neurabin and RGS4 reveals that $\mathrm{A} 1 \mathrm{R}$ and RGS4 interact with overlapping sequences of neurabin (Fig. 3). Thus, to form a ternary complex (as shown in Fig. 4B), A1R and RGS4 likely each interact with one neurabin molecule in a neurabin oligomer. Indeed, neurabin domains that are required for oligomerization are essential for formation of the A1R/neurabin/RGS4 ternary complex (Fig. 5C). Despite its ability to interact with A1R and RGS4 individually, the truncated neurabin mutant that cannot oligomerize (Nrb1-597) fails to stabilize plasma membrane localization of RGS4 (Fig. 6C) and is unable to attenuate A1R signaling (Fig. 7A). Taken together, these data reveal a novel mode of regulation of GPCR signaling by scaffolding proteins, specifically the formation of a ternary complex with the receptor and a regulator through homo-oligomerization (Fig. 5A).

Scaffolding proteins play crucial roles in the regulation of GPCR trafficking and signaling (Romero et al., 2011; Walther and Ferguson, 2015). They can target receptors to specific subcellular compartments, determine the endocytic route of the receptor, and link the receptor to specific effectors and regulators. Although a number of scaffolding proteins are predicted to have oligomerization domains (Hu et al., 2015), the significance of oligomerization of these proteins in GPCR regulation remains largely elusive, except for homer proteins. The long forms of homer proteins form dimers and tetramers through the $\mathrm{C}$-terminal coiled-coil domains (Hayashi et al., 2009). The resulting hub structure connects synaptic proteins and signaling components and targets the group I metabotropic glutamate receptors to postsynaptic
A
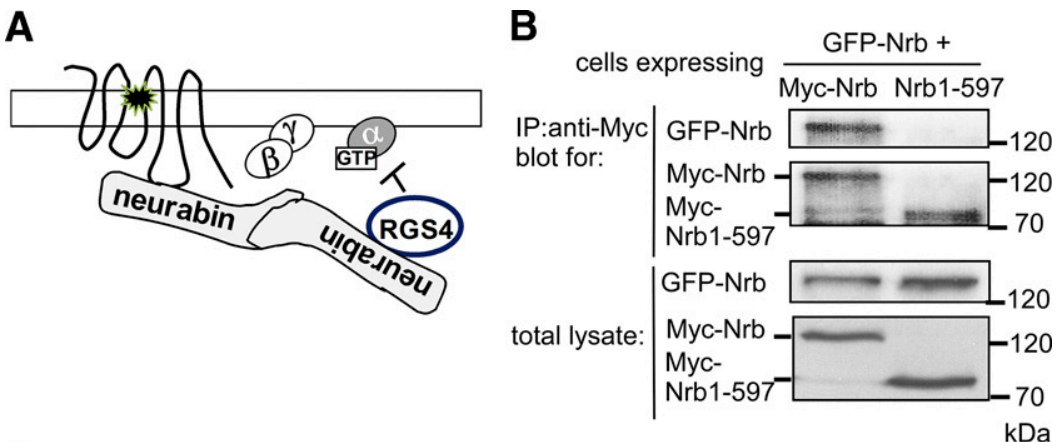

C

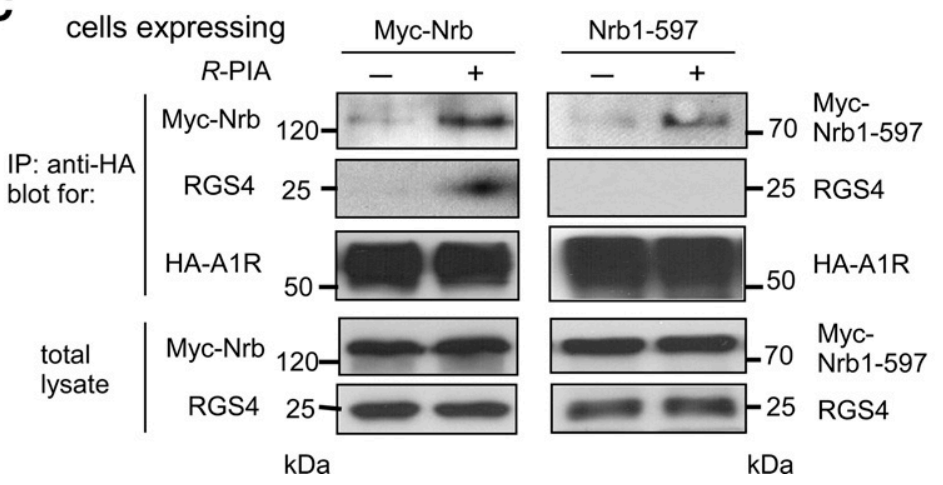

Fig. 5. Truncated neurabin lacking coiled-coil and SAM domains failed to scaffold formation of the A1R-RGS4 complex. (A) A working model for A1R/neurabin/RGS4 complex formation. (B) Truncated neurabin lacking coiledcoil and SAM domains did not form oligomers. HEK293 cells coexpressing GFP-Nrb with Myc-Nrb or GFP-Nrb1-597 with Myc-Nrb1-597 were lysed and subjected to the IP assay using an anti-Myc antibody. Blots representative of at least three independent experiments are shown. (C) No complex formation between A1R and RGS4 was detected upon $R$-PIA stimulation in cells expressing Nrb1-597. HEK293 cells coexpressing HA-A1R, Myc-Nrb1-597, and RGS4 were stimulated with $R$-PIA $(1 \mu \mathrm{M})$ for 5 minutes. Cell lysates were subjected to IP assay using an anti-Myc antibody and analyzed by Western blot. Blots representative of four independent experiments are shown. 
A
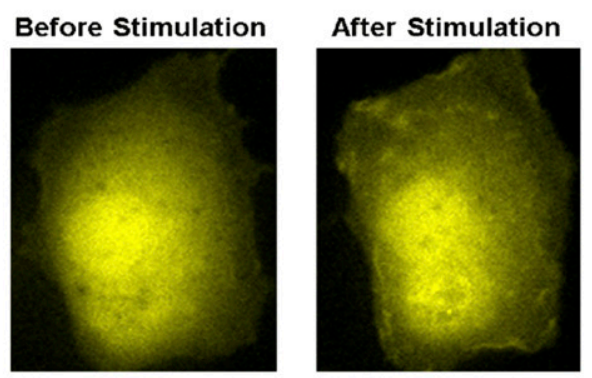

B Full Length Neurabin

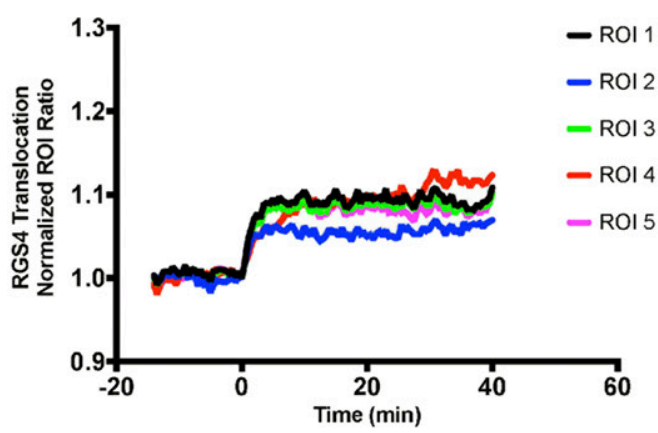

C

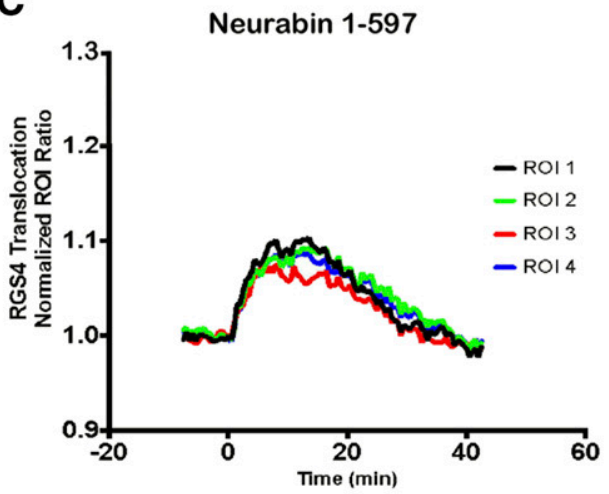

D

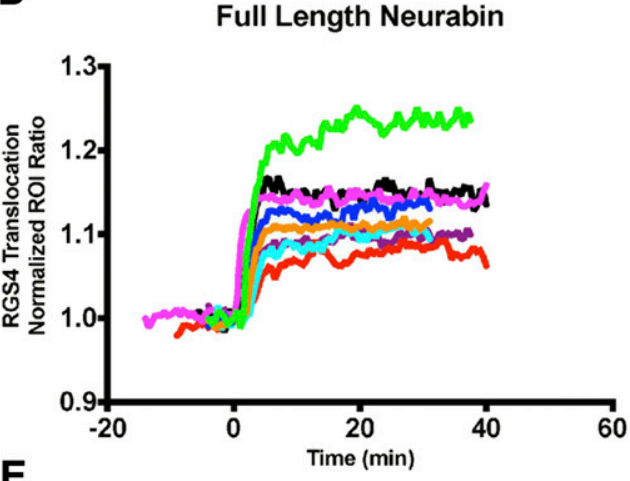

E

Neurabin 1-597

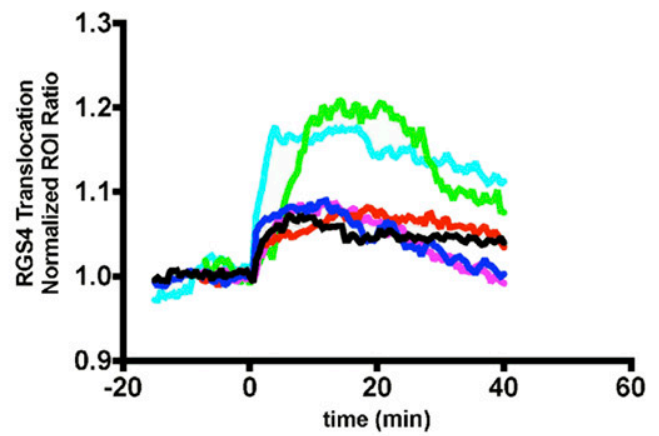

$\mathbf{F}$

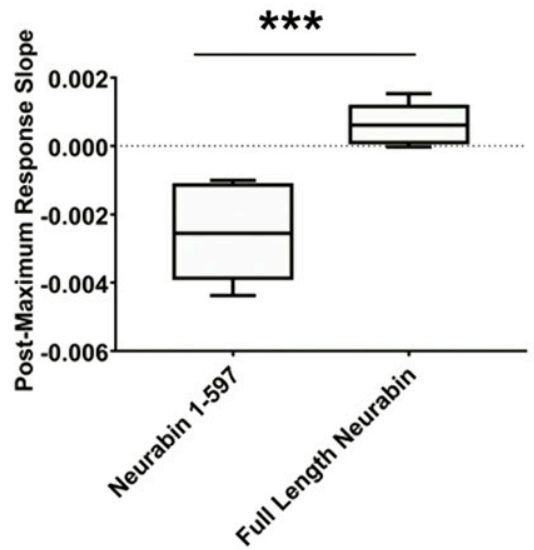

Fig. 6. A1R activation led to sustained localization of RGS4 at the plasma membrane of live cells with expression of full-length neurabin but failed to do so when Nrb1-597 was expressed. (A) Representative images of RGS4 localization before and after $1 \mu \mathrm{M} R$-PIA stimulation in Cos7 cells coexpressing A1R-CFP and RGS4-YFP with full-length neurabin. (B) Representative traces of RGS4 showing sustained RGS4 translocation within different plasma membrane ROIs in a single Cos7 cell with full-length neurabin coexpressed with A1R-CFP and RGS4-YFP $(n=6)$. $R$-PIA $(1 \mu \mathrm{M})$ was added at $t=0$. (C) Representative traces showing transient RGS4 translocation within different plasma membrane ROIs in a single Cos7 cell with Nrb1-597 coexpressed with A1R-CFP and RGS4-YFP $(n=4)$. R-PIA $(1 \mu \mathrm{M})$ was added at $t=0$. (D) Multiple translocation traces from different cells expressing the full-length neurabin demonstrate uniform responses of sustained RGS4 localization at the plasma membrane across the cells; $n=8$. (E) Multiple traces from different cells expressing Nrb1-597 demonstrate nonuniform responses of RGS4 translocation across the cells with a general transient nature $(n=6)$. (F) The sustained plasma membrane localization of RGS4 was analyzed by comparing the slope of the trace after the maximal response in each cell. $* * * P<0.001$ between the slopes of cells in the presence of full-length neurabin and Nrb1-597A.

compartments and their effectors (Bockaert et al., 2010). To our knowledge, our study provides the first example of oligomerization being required for a scaffolding protein to link the receptor to a RGS protein to modulate receptor signaling strength. In addition to forming homo-oligomers, neurabin can form hetero-oligomers with its homolog, spinophilin (Nakanishi et al., 1997; MacMillan et al., 1999; Oliver et al., 2002). Since spinophilin does not interact with A1R, neurabinspinophilin oligomerization in the postsynaptic compartments may reduce the ability of neurabin to attenuate $A 1 R$ responses, adding another level of complexity in A1R regulation.

We previously demonstrated that neurabin specifically links RGS4, but not its close homolog RGS2, to A1R signaling (Chen et al., 2012). RGS4 is highly expressed in the brain (Larminie et al., 2004) and regulates multiple Gq or Gi-coupled receptors to modulate synaptic signaling and plasticity (Gerber et al., 2016). Plasma membrane localization of RGS proteins is critical for their ability to terminate $\mathrm{G}$ protein signaling (Hollinger and Hepler, 2002; 
A

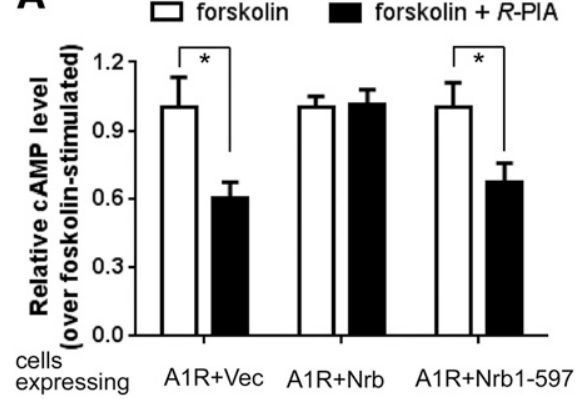

B

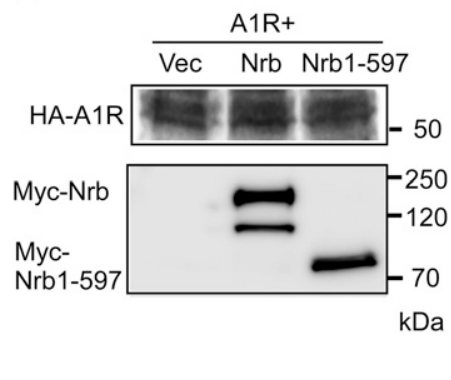

Fig. 7. Expression of Nrb1-597 failed to cause attenuation of A1R signaling. (A) Expression of Nrb1-597 had no effect on A1R-mediated inhibition of cAMP production. CHO-K1 cells expressing HA-A1R alone (plus empty vector) or in combination with either neurabin wild type or Nrb1-597 were treated with $10 \mu \mathrm{M}$ forskolin alone or forskolin plus $1 \mu \mathrm{M} R$-PIA. The average cAMP concentration at the basal level in cells was $3.45 \times 10^{-9} \mathrm{M}$ and became $3.89 \times 10^{-8} \mathrm{M}$ with forskolin stimulation (i.e., forskolin alone). Data are expressed as the fold change in cAMP production over forskolin-alone control (defined as 1.0-fold) ( $n=6$ for cells expressing the A1R alone, $n=8$ for cells expressing the A1R and neurabin, $n=7$ for cells expressing A1R and Nrb1-597). $* P<0.05$ ( $R$-PIA stimulated vs. control by $t$ test). (B) Representative Western blots showing expression of HA-A1R, Nrb, and Nrb1-597 in the same preparation of cells analyzed for cAMP production. Note that a degradation band of full-length Nrb was detected (by Myc antibody) after the assay sample preparation. Vec, vehicle.

Kach et al., 2012). RGS4 is mainly localized in the cytosol and could be recruited to the plasma membrane through interactions with GPCRs or $\mathrm{G} \alpha$ proteins (Roy et al., 2003). Using live-cell imaging, we demonstrate that RGS4 rapidly translocates to the plasma membrane upon A1R stimulation (Figs. 2 and 7), likely through direct interaction with the receptor or $\mathrm{G}$ proteins. In cells lacking neurabin expression or expressing the mutant neurabin Nrb1-597, plasma membrane localization of RGS4 is transient (Figs. 2 and 7) and A1Rmediated cAMP inhibition is not affected (Fig. 7). On the other hand, when the full-length neurabin is present, plasma membrane localization of RGS4 is prolonged (Figs. 2 and 7) and A1R signaling is attenuated (Fig. 7). These results suggest that although neurabin is not required for the initial membrane recruitment of RGS4, neurabin is essential for the sustained localization of RGS4 at the plasma membrane and subsequent attenuation of A1R signaling.

We and others have shown that A1R does not undergo rapid phosphorylation after agonist stimulation (Palmer et al., 1996; Chen et al., 2012), which is often a prerequisite for arrestin binding to and desensitization of many GPCRs. Consistently, desensitization of A1R signaling takes place at a much slower pace than many GPCRs, including its homologs adenosine A2R and A3R (Ramkumar et al., 1991; Palmer et al., 1996; Wetherington and Lambert, 2002). Collectively, our data suggest that the prolonged membrane recruitment of RGS4, which is scaffolded by neurabin, represents a critical mechanism for finetuning A1R signaling. In addition, A1R activation has been reported to reduce M2 muscarinic acetylcholine receptor responsiveness in neurons (Shakirzyanova et al., 2006). The A1Rinduced, sustained RGS4 membrane localization revealed in our study may contribute to the cross-desensitization of M2 muscarinic receptor signaling after $\mathrm{A} 1 \mathrm{R}$ agonist stimulation.

In summary, this study reveals that homo-oligomerization of neurabin is required for stabilizing RGS4 on the plasma membrane to attenuate A1R signaling. Our mechanistic analysis of neurabin-mediated regulation of A1R signaling provides valuable information for the future development of therapeutic interventions targeting $\mathrm{A} 1 \mathrm{R}$ in the nervous system.

\section{Acknowledgments}

The authors thank Dr. Christopher Cottingham (Morehead State University, Morehead, KY) for critical reading and helpful editing of the manuscript.

\section{Authorship Contributions}

Participated in research design: Chen, Booth, Zachariou, Jiao, Zhang, Q. Wang.

Conducted experiments: Chen, Booth, H. Wang, R.X. Wang, Terzi. Contributed new reagents or analytic tools: Booth, Zhang.

Performed data analysis: Chen, Booth, Jiao, Zhang, Q. Wang.

Wrote or contributed to the writing of the manuscript: Chen, Booth, Zachariou, Jiao, Zhang, Q. Wang.

\section{References}

Bockaert J, Perroy J, Bécamel C, Marin P, and Fagni L (2010) GPCR interacting proteins (GIPs) in the nervous system: roles in physiology and pathologies. Annu Rev Pharmacol Toxicol 50:89-109.

Boison D (2008) Adenosine as a neuromodulator in neurological diseases. Curr Opin Pharmacol 8:2-7.

Burnett PE, Blackshaw S, Lai MM, Qureshi IA, Burnett AF, Sabatini DM, and Snyder SH (1998) Neurabin is a synaptic protein linking p70 S6 kinase and the neuronal cytoskeleton. Proc Natl Acad Sci USA 95:8351-8356.

Chen H and Lambert NA (2000) Endogenous regulators of G protein signaling proteins regulate presynaptic inhibition at rat hippocampal synapses. Proc Natl Acad Sci USA 97:12810-12815.

Chen Y, Liu Y, Cottingham C, McMahon L, Jiao K, Greengard P, and Wang Q (2012) Neurabin scaffolding of adenosine receptor and RGS4 regulates anti-seizure effect of endogenous adenosine. J Neurosci 32:2683-2695.

Chen Y, Peng Y, Che P, Gannon M, Liu Y, Li L, Bu G, van Groen T, Jiao K, and Wang $\mathrm{Q}(2014) \alpha(2 \mathrm{~A})$ adrenergic receptor promotes amyloidogenesis through disrupting APP-SorLA interaction. Proc Natl Acad Sci USA 111:17296-17301.

Cunha RA (2001) Adenosine as a neuromodulator and as a homeostatic regulator in the nervous system: different roles, different sources and different receptors. Neurochem Int 38:107-125.

Dunwiddie TV and Masino SA (2001) The role and regulation of adenosine in the central nervous system. Annu Rev Neurosci 24:31-55.

Fu Y, Huang X, Zhong H, Mortensen RM, D’Alecy LG, and Neubig RR (2006) Endogenous RGS proteins and Galpha subtypes differentially control muscarinic and adenosine-mediated chronotropic effects. Circ Res 98:659-666.

Gerber KJ, Squires KE, and Hepler JR (2016) Roles for regulator of G protein signaling proteins in synaptic signaling and plasticity. Mol Pharmacol 89:273-286.

Haas HL and Selbach O (2000) Functions of neuronal adenosine receptors. Naunyn Schmiedebergs Arch Pharmacol 362:375-381.

Hayashi MK, Tang C, Verpelli C, Narayanan R, Stearns MH, Xu RM, Li H, Sala C, and Hayashi Y (2009) The postsynaptic density proteins Homer and Shank form a polymeric network structure. Cell 137:159-171.

Hollinger S and Hepler JR (2002) Cellular regulation of RGS proteins: modulators and integrators of G protein signaling. Pharmacol Rev 54:527-559.

Hu J, Neiswinger J, Zhang J, Zhu H, and Qian J (2015) Systematic prediction of scaffold proteins reveals new design principles in scaffold-mediated signal transduction. PLoS Comput Biol 11:e1004508.

Kach J, Sethakorn N, and Dulin NO (2012) A finer tuning of G-protein signaling through regulated control of RGS proteins. Am J Physiol Heart Circ Physiol 303: H19-H35.

Larminie C, Murdock P, Walhin JP, Duckworth M, Blumer KJ, Scheideler MA, and Garnier M (2004) Selective expression of regulators of G-protein signaling (RGS) in the human central nervous system. Brain Res Mol Brain Res 122:24-34.

MacMillan LB, Bass MA, Cheng N, Howard EF, Tamura M, Strack S, Wadzinski BE, and Colbran RJ (1999) Brain actin-associated protein phosphatase 1 holoenzymes containing spinophilin, neurabin, and selected catalytic subunit isoforms. J Biol Chem 274:35845-35854.

Nakanishi H, Obaishi H, Satoh A, Wada M, Mandai K, Satoh K, Nishioka H, Matsuura Y, Mizoguchi A, and Takai Y (1997) Neurabin: a novel neural 
tissue-specific actin filament-binding protein involved in neurite formation. $J$ Cell Biol 139:951-961.

Neitzel KL and Hepler JR (2006) Cellular mechanisms that determine selective RGS protein regulation of $\mathrm{G}$ protein-coupled receptor signaling. Semin Cell Dev Biol 17: $383-389$.

Neubig RR and Siderovski DP (2002) Regulators of G-protein signalling as new central nervous system drug targets. Nat Rev Drug Discov 1:187-197.

Oliver CJ, Terry-Lorenzo RT, Elliott E, Bloomer WA, Li S, Brautigan DL, Colbran RJ, and Shenolikar S (2002) Targeting protein phosphatase 1 (PP1) to the actin cytoskeleton: the neurabin I/PP1 complex regulates cell morphology. Mol Cell Biol 22:4690-4701.

Palmer TM, Benovic JL, and Stiles GL (1996) Molecular basis for subtype-specific desensitization of inhibitory adenosine receptors. Analysis of a chimeric A1-A3 adenosine receptor. J Biol Chem 271:15272-15278.

Ramkumar V, Olah ME, Jacobson KA, and Stiles GL (1991) Distinct pathways of desensitization of A1- and A2-adenosine receptors in DDT1 MF-2 cells. Mol Pharmacol 40:639-647.

Romero G, von Zastrow M, and Friedman PA (2011) Role of PDZ proteins in regulating trafficking, signaling, and function of GPCRs: means, motif, and opportunity. Adv Pharmacol 62:279-314.

Ross EM and Wilkie TM (2000) GTPase-activating proteins for heterotrimeric G proteins: regulators of G protein signaling (RGS) and RGS-like proteins. Annu Rev Biochem 69:795-827.

Roy AA, Lemberg KE, and Chidiac P (2003) Recruitment of RGS2 and RGS4 to the plasma membrane by $\mathrm{G}$ proteins and receptors reflects functional interactions. $\mathrm{Mol}$ Pharmacol 64:587-593.
Sebastião AM and Ribeiro JA (2009) Adenosine receptors and the central nervous system. Handb Exp Pharmacol 193:471-534.

Shakirzyanova AV, Bukharaeva EA, Nikolsky EE, and Giniatullin RA (2006) Negative cross-talk between presynaptic adenosine and acetylcholine receptors. Eur $J$ Neurosci 24:105-115.

Sjögren B and Neubig RR (2010) Thinking outside of the "RGS box": new approaches to therapeutic targeting of regulators of $\mathrm{G}$ protein signaling. Mol Pharmacol $\mathbf{7 8}$ $550-557$.

Stone TW (2002) Purines and neuroprotection. Adv Exp Med Biol 513:249-280.

Walther C and Ferguson SS (2015) Minireview: role of intracellular scaffolding proteins in the regulation of endocrine $\mathrm{G}$ protein-coupled receptor signaling. $\mathrm{Mol}$ Endocrinol 29:814-830.

Wang Q and Limbird LE (2002) Regulated interactions of the alpha 2A adren ergic receptor with spinophilin, 14-3-3zeta, and arrestin 3. J Biol Chem 277 50589-50596.

Wetherington JP and Lambert NA (2002) Differential desensitization of responses mediated by presynaptic and postsynaptic A1 adenosine receptors. $J$ Neurosci 22 : 1248-1255

Xie GX and Palmer PP (2007) How regulators of G protein signaling achieve selective regulation. J Mol Biol 366:349-365.

Address correspondence to: Qin Wang, Departments of Cell, Developmental, and Integrative Biology, University of Alabama, 1918 University Blvd., 986 MCLM, Birmingham, AL 35294. E-mail: qinwang@uab.edu 